\title{
Efectos de la suplementación con vitamina E en la reducción del dolor muscular de inicio retardado. Una revisión narrativa
}

DOI: 10.17533/udea.penh.v21n2a07

PERSPECTIVAS EN NUTRICIÓN HUMANA

ISSN 0124-4108

Escuela de Nutrición y Dietética, Universidad de Antioquia. Medellín, Colombia

Vol. 21, N. ${ }^{\circ}$ 2, julio-diciembre de 2019, pp. 219-227.
Artículo recibido: 31 de diciembre de 2018

Aprobado: 4 de diciembre de 2019

\section{Héctor Fuentes-Barría*; Raúl Aguilera-Eguía; Catalina González-Wong³; Aylinne Schramm-Saavedra4; Denisse Muñoz-Peña ${ }^{5}$}

\section{- - - Resumen}

Antecedentes: actualmente existe un creciente interés en investigar métodos que logren disminuir el dolor muscular de inicio retardado. Se ha postulado que antioxidantes como las vitaminas $C$ y E pueden atenuarlo. Objetivo: evaluar la efectividad de vitamina $E$ en la atenuación del dolor muscular de inicio retardado. Materiales y métodos: se realizó una búsqueda bibliográfica en las bases de datos Medline, Registro Central Cochrane, Scopus, SportDiscus y SciELO, utilizando los términos: "vitamin e supplementation", "muscle damage" y "delayed onset muscle soreness". Resultados: se revisaron estudios en diversos grupos poblacionales, con diferentes métodos de evaluación y diversas dosis de vitamina $E$. Los resultados obtenidos fueron controversiales, en algunos casos se demostró un efecto atenuante del dolor muscular de inicio retardado y en otros casos no. Conclusiones: la vitamina $\mathrm{E}$ produce una disminución del estrés oxidativo y estabilización el sarcolema; no obstante, faltan pruebas concluyentes para afirmar que la vitamina $E$ tenga el efecto de generar mejoras en los biomarcadores asociados al dolor muscular de inicio retardado.

Palabras clave: suplementos dietéticos, vitamina E, tocoferoles, antioxidantes, dolor muscular.

$1^{*} \quad$ Autor de correspondencia. Magíster en Ciencias de la Actividad Física y Deportes Aplicadas al Entrenamiento, Rehabilitación y Reintegro Deportivo, Facultad de Salud Universidad Santo Tomas, Chile. h3ct0r.fuentes.b@gmail.com ORCID: orcid.org/0000-0003-0774-0848

2 Departamento de Salud Pública, Facultad de Medicina, Carrera de Kinesiología. Universidad Católica de la Santísima Concepción. Concepción, Chile. ORCID: orcid.org/0000-0002-4123-4255

3 Magíster de Gestión en Salud, Facultad de Salud Universidad del Desarrollo, Chile. ORCID: orcid.org/0000-0003-0360-8567

4 Nutrición y Dietética, Facultad de Medicina, Universidad Finis Terrae, Chile.

5 Nutrición y Dietética, Facultad de Ciencias de la Salud, Universidad Autónoma de Chile, Chile.

Cómo citar este artículo: Fuentes-Barría H, Aguilera-Eguía R, González-Wong C, Schramm-Saavedra A, Muñoz-Peña D. Efectos de la suplementación con vitamina $\mathrm{E}$ en la reducción del dolor muscular de inicio retardado. Una revisión narrativa. Perspect Nutr Humana. 2019;21:219-227. DOI: 10.17533/udea.penh.v21n2a07 
Efectos de la suplementación con vitamina $\mathrm{E}$

\section{Effects of Vitamin E Supplementation in Reducing Delayed Onset Muscle Pain. A Narrative Review}

\section{Abstract}

Background: Currently, there is a growing interest in investigating methods that reduce delayed onset muscle pain. It has been postulated that antioxidants such as vitamin $\mathrm{C}$ and $\mathrm{E}$ can attenuate it. Objective: To evaluate the effectiveness of vitamin $\mathrm{E}$ in mitigating delayed onset muscle pain. Materials and Methods: A bibliographic search was carried out in the following databases: Medline, Registry Central Cochrane, Scopus, SportDiscus and SciELO, using the terms: "vitamin e supplementation", "muscle damage" and "delayed onset muscle soreness". Results: Studies in diverse population groups were reviewed, with various evaluation methods and differing doses of vitamin $\mathrm{E}$. The results were very controversial, in some cases a delayed onset pain effect was demonstrated and in other cases not. Conclusion: Vitamin E produces a decrease in oxidative stress and stabilization of sarcolemma, however, conclusive evidence is lacking to state that vitamin $\mathrm{E}$ will have the effect of generating improvements in biomarkers associated with delayed onset muscle pain.

Keywords: Dietary supplements, vitamin E, tocopherols, antioxidants, muscle soreness.

\section{INTRODUCCIÓN}

El dolor muscular de inicio retardado (DOMS, por sus siglas en inglés) se clasifica como una lesión por mecanismo de distensión muscular de grado 1 , el cual suele presentarse de 24 a 72 horas luego del ejercicio y desaparece entre cinco y siete días después de la actividad (1-3). El DOMS se caracteriza por presentar síntomas somáticos como dolor, rigidez, hipersensibilidad e inflamación muscular, a menudo asociados con desacondicionamiento físico o ejercicios de tipo excéntrico de alta intensidad (1-5).

La etiología del DOMS ha considerado la posible participación de factores como inflamación, espasmo muscular, aumento de la temperatura muscular, incremento de la producción de radicales libres y daño del tejido muscular o conectivo. En particular, se ha postulado la teoría del daño muscular inducido por múltiples factores como posible causa del DOMS (2). Actualmente, la literatura destaca entre los tratamientos no farmacológicos la suplementación nutricional de flavonoides,
L-carnitina y de vitaminas como C, D y E (3,6-10). Esta última se caracteriza por estar conformada por un grupo genérico de ocho compuestos, los cuales se subdividen en $\alpha, \beta, \gamma \delta$-tocoferoles y $\alpha, \beta, \gamma \delta$-tocotrienoles. En los seres humanos, se encuentra en la forma de $\alpha$-tocoferol y las mejores fuentes alimentarias son los vegetales de hoja verde, cítricos, frutas, legumbres, nueces, semillas y aceites (2,11-13).

La vitamina $E$ se ha relacionado con un posible papel potencial en la atenuación de ciertos signos y síntomas como el DOMS y estrés oxidativo, pero no con la reducción del daño muscular $(2,8,14)$. Por esta razón, en la presente revisión se planteó como objetivo evaluar la efectividad de la vitamina $E$ en la atenuación del DOMS.

\section{MATERIALES Y MÉTODOS}

Se realizó una revisión narrativa de la literatura por medio de la búsqueda de artículos en las siguientes bases de datos: Mediline, Cochrane Library, Scopus, SportDiscus y SciELO. La estrategia 
de búsqueda se elaboró utilizando los términos "vitamin e supplementation", "muscle damage", "delayed onset muscle soreness" en forma conjunta con los operadores booleano "AND" "OR", con la finalidad de obtener resultados concretos y dirigidos al objetivo de estudio. La búsqueda bibliográfica estuvo limitada al título, resumen y palabras clave en artículos publicados desde el 2000 hasta el 2018.

A los artículos seleccionados por la búsqueda preliminar se les aplicó un filtro a partir de una lectura crítica de los títulos o resúmenes; se excluyeron todos los artículos duplicados y los seleccionados se analizaron bajo los siguientes criterios: artículos originales, realizados en humanos de ambos sexos, que estudien solo la suplementación de vitamina $E$ y su relación directa con el DOMS o con biomarcadores de estrés y daño oxidativo, como malonildialdehido (MDA), ácido tiobarbitúrico (TBA), indicadores de daño muscular como creatina kinasa (CK) y lactato deshidrogenasa (LDH) comparado con placebo. Por último, esta revisión referencia 50 artículos de interés con el objetivo de estudio, de los cuales se analizaron seis.

\section{RESULTADOS}

La mayoría de los estudios referentes al DOMS y biomarcadores de estrés oxidativo se ha relacionado con la suplementación nutricional de vitamina $E$ en forma individual o combinada con vitamina $C(15-22)$.

La tabla 1 resume los aspectos metodológicos de los seis artículos seleccionados (23-28), con relación a sus protocolos de suplementación de vitamina $E$. Todos los estudios suministraron vitamina $E$ en forma de cápsulas en dosis que iban desde 250 mg/día (375 UI) hasta 800 mg (1200 Ul/día), durante un periodo que osciló entre $1 \mathrm{~h}$ hasta 12 semanas. Por otro lado, la tabla 2 resume las principales medidas de resultados y las conclusiones de los seis artículos analizados (23-28). Tres estudios analizaron el DOMS y otras variables de daño celular (23-25), mientras que los tres artículos restantes analizaron el estrés oxidativo en conjunto con otras variables de daño celular (26-28).

Los estudios que analizaron el DOMS utilizaron la escala visual análoga (VAS), mientras que los

Tabla 1. Suplementación de vitamina E en los estudios analizados

\begin{tabular}{|c|c|c|c|}
\hline Autor y año & $\begin{array}{c}\text { Muestra } \\
\text { (vit } \mathrm{E} / \text { placebo) }\end{array}$ & Dosis & $\begin{array}{l}\text { Tiempo de suplementación } \\
\text { (preejercicio/posejercicio) }\end{array}$ \\
\hline Silva, 2010 (23) & $21(11 / 10)$ & $\begin{array}{c}\text { cápsula } \\
\text { (800 Ul/día) }\end{array}$ & 21 días (14 /7) \\
\hline Avery, 2003 (24) & $18(9 / 9)$ & $\begin{array}{c}\text { cápsula } \\
\text { (1200 Ul/día) }\end{array}$ & 31 días $(21 / 10)$ \\
\hline Beaton, 2002 (25) & $16(9 / 7)$ & $\begin{array}{c}\text { cápsula } \\
\text { (1200 Ul/día) }\end{array}$ & 30 días \\
\hline Itoh, 2000 (26) & $14(7 / 7)$ & $\begin{array}{c}\text { cápsula } \\
\text { (1200 Ul/día) }\end{array}$ & $\begin{array}{l}4 \text { semanas y } 6 \text { días } \\
\text { (4 semanas / } 6 \text { días) }\end{array}$ \\
\hline Sacheck, 2003 (27) & $32(16 / 16)$ & $\begin{array}{c}\text { cápsula } \\
\text { (1000 Ul/día) }\end{array}$ & 12 semanas \\
\hline Santos, 2016(28) & 9 & $\begin{array}{c}\text { cápsula } \\
\text { (375 mg/día) }\end{array}$ & $1 \mathrm{~h}$ \\
\hline
\end{tabular}


Efectos de la suplementación con vitamina $\mathrm{E}$

Tabla 2. Resumen de las medidas de resultado en los estudios incluidos

\begin{tabular}{|c|c|c|c|c|c|}
\hline $\begin{array}{l}\text { Autor principal } \\
\text { y año }\end{array}$ & Indicadores & $\begin{array}{l}\text { Variables } \\
\text { estudiadas }\end{array}$ & Ejercicio & $\begin{array}{l}\text { Evaluación } \\
\text { posejercicio }\end{array}$ & $\begin{array}{c}\text { Efecto de la suplementación } \\
\text { con vitamina } E\end{array}$ \\
\hline \multirow{4}{*}{ Silva, 2010 (23) } & DOMS biceps & $\operatorname{VAS}(0$ a $10 \mathrm{~cm})$ & \multirow{4}{*}{$\begin{array}{l}3 \text { series isocinéticas } \\
\text { hasta la fatiga }\end{array}$} & \multirow{4}{*}{48,96 y $168 \mathrm{~h}$} & \multirow{4}{*}{$\begin{array}{l}\text { La suplementación con } \\
\text { vitamina } E \text { atenúa el dolor } \\
\text { muscular, el daño muscular y } \\
\text { el estrés oxidativo, pero no la } \\
\text { inflamación }\end{array}$} \\
\hline & Daño celular & $\mathrm{LDH}$ & & & \\
\hline & Estrés oxidativo & $\begin{array}{l}\text { Peroxidación lipí- } \\
\text { dica, carbonilación } \\
\text { de proteína }\end{array}$ & & & \\
\hline & Inflamación & TNF- $\alpha$ e IL-10 & & & \\
\hline Avery, 2003 (24) & $\begin{array}{l}\text { DOMS hombro y } \\
\text { cadera } \\
\text { Estrés oxidativo } \\
\text { Daño celular }\end{array}$ & $\begin{array}{l}\text { VAS }(0 \text { a } 10 \mathrm{~cm}) \\
\text { MDA } \\
\text { CK }\end{array}$ & $\begin{array}{l}4 \times 10 \text { contraccio- } \\
\text { nes concéntrica } \\
\text { excéntrica en } \\
\text { brazos y piernas }\end{array}$ & 24,48 y $72 h$ & $\begin{array}{l}\text { La suplementación con } \\
\text { vitamina E no mostró efecto } \\
\text { sobre el DOMS, el daño mus- } \\
\text { cular ni el estrés oxidativo }\end{array}$ \\
\hline Beaton, 2002 (25) & DOMS cuádriceps & $\operatorname{VAS}(0$ a $10 \mathrm{~cm})$ & $\begin{array}{l}24 \times 10 \text { acciones } \\
\text { excéntricas }\end{array}$ & 24 y $48 \mathrm{~h}$ & $\begin{array}{l}\text { La suplementación con } \\
\text { vitamina E no mostró efecto } \\
\text { sobre DOMS ni sobre el daño } \\
\text { muscular no inflamatorio }\end{array}$ \\
\hline Itoh, 2000 (26) & $\begin{array}{l}\text { Daño muscular } \\
\text { Daño oxidativo }\end{array}$ & $\begin{array}{l}\text { CK y LDH } \\
\text { TBH }\end{array}$ & $\begin{array}{l}\text { Contracción } \\
\text { concéntrica excén- } \\
\text { trica en carrera }\end{array}$ & $\begin{array}{l}24 \text { h y } 3 \\
\text { semanas }\end{array}$ & $\begin{array}{l}\text { La suplementación con } \\
\text { vitamina E atenúa el daño } \\
\text { muscular y el estrés oxidativo }\end{array}$ \\
\hline Sacheck, 2003 (27) & $\begin{array}{l}\text { Estrés oxidativo } \\
\text { Capacidad } \\
\text { antioxidante } \\
\text { Daño muscular }\end{array}$ & $\begin{array}{c}\text { MDA e iPF2 } \alpha \\
\text { Absorción de radi- } \\
\text { cales de oxígeno } \\
\text { CK }\end{array}$ & $\begin{array}{l}45 \text { min acciones } \\
\text { excéntricas en } \\
\text { carrera }\end{array}$ & $0,6,24$ y $72 \mathrm{~h}$ & $\begin{array}{l}\text { La suplementación con vita- } \\
\text { mina E disminuyó el estrés } \\
\text { oxidativo y el daño muscular }\end{array}$ \\
\hline Santos, 2016 (28) & $\begin{array}{l}\text { Indicadores de } \\
\text { daño muscular } \\
\text { Indicadores de } \\
\text { inflamación }\end{array}$ & $\begin{array}{l}\text { IL-6, TNF- } \alpha, \\
\text { IL-1. }{ }^{a} \text { e IL-10 }\end{array}$ & $\begin{array}{l}1 \mathrm{~h} \text { aeróbicos en } \\
\text { hipoxia }\end{array}$ & $0 \mathrm{y} 1 \mathrm{~h}$ & $\begin{array}{c}\text { La suplementación con } \\
\text { vitamina E redujo el daño } \\
\text { muscular, el estrés oxidativo } \\
\text { y la inflamación }\end{array}$ \\
\hline
\end{tabular}

CK: creatina quinasa, LDH: lactato deshidrogenasa, IL: Interleucina, MDA: malondialdehyde, DOMS: dolor muscular de inicio retardado, TNF- $\alpha$ : factor de necrosis tumoral $\alpha$, iPF2: $\alpha$ isoprostano F2 $\alpha$, ácido tiobarbitúrico.

artículos que analizaron el estrés oxidativo, daño celular e inflamación utilizaron los biomarcadores MDA, CK, LDH y IL. Los tres estudios relacionados con el DOMS prescribieron ejercicios isocinéticos, excéntricos y concéntricos/excéntricos de corta duración, mientras que los tres estudios relacionados con el análisis del estrés oxidativo prescribieron ejercicio aeróbico de tipo excéntrico, aeróbicos en condiciones hipóxicas y aeróbicos con fase excéntrica/concéntrica.

Con relación a los principales resultados, de los tres estudios que analizaron el DOMS (23-25), solo uno reportó una pequeña disminución en la percepción del DOMS (23), mientras que en los dos estudios restantes no se observaron efectos positivos relacionados con la suplementación de vitamina $\mathrm{E}$ y DOMS comparado con placebo $(24,25)$. Por otro lado, los tres estudios que analizaron el efecto de la vitamina $E$ sobre el estrés oxidativo reportaron cambios favorables en los respectivos biomarcadores (26-28). 


\section{DISCUSIÓN}

Los resultados observados son contradictorios; Silva et al. (23), al comparar una ingesta durante 14 días antes y 7 días después de los ejercicios isocinéticos hasta la fatiga, reportó una atenuación del dolor muscular, del daño muscular y del estrés oxidativo, pero no la inflamación.

No obstante, Avery et al. (24), al analizar los efectos de la vitamina $E$ sobre la recuperación de los brazos y piernas, determinó que una suplementación de 1200 UI/día no mostró efecto sobre el DOMS, el daño muscular ni el estrés oxidativo, mientras que Beaton et al. (25), al suministrar la misma dosis 30 días antes de realizar acciones excéntricas, encontró que no hay efecto sobre DOMS ni daño muscular inflamatorio.

Estos resultados pueden explicarse debido a que el ejercicio físico tanto de tipo aeróbico como anaeróbico puede provocar un desbalance en la homeostasis oxidante y antioxidante. En favor de los primeros, este desbalance o estrés oxidativo induce una ruptura del proceso de control y señalización fisiológica del sistema de reducción oxidación (REDOX) y posteriormente un daño celular reflejado en un aumento de CK y LD, marcadores de estrés oxidativo como el MDA y citoquininas proinflamatorias TNF- $\alpha$ y IL-6, (2,29-33).

Siguiendo esta argumentación, Itoh et al. (26) determinaron que una suplementación de $1200 \mathrm{UI} /$ día durante 4 semanas antes de una carrera de resistencia a intensidad máxima puede reducir la pérdida de CK y LDH, indicadores de daño muscular, a causa del estrés oxidativo luego de 6 días consecutivos de carreras.

Del mismo modo, Sacheck et al. (27) sugieren cambios moderados del estrés oxidativo inducido por el ejercicio excéntrico, independiente de la edad, mientras que Santos et al. (28), luego de suministrar $250 \mathrm{mg}$ (375 Ul/día) de suplementos de vitamina $E$ una hora antes de ejercicios en condición hipóxica, reportaron cambios favorables en los marcadores de daño celular y concentración de citoquímicas proinflamatorias.

Estos resultados permiten corroborar que la vitamina $E$ solo tiene un efecto comprobado sobre la capacidad antioxidante y la disminución de la peroxidación lipídica; sin embargo, estos efectos dependerán de múltiples factores intrínsecos y extrínsecos, como nivel de entrenamiento, fatiga, intensidad de la carga de trabajo, duración del ejercicio, edad y el sexo de cada individuo (29-40).

El hecho de que no se haya comprobado una función reguladora del DOMS por parte de la vitamina $\mathrm{E}$ se puede atribuir al tiempo de exposición, debido a que se ha observado que intervenciones agudas no siempre logran reflejar disminuciones en biomarcadores sanguíneos como la CK $(41,42)$.

Siguiendo esta línea, Burton et al. (43) observaron una disminución en la concentración sérica de vitamina $\mathrm{E}$ de hasta aproximadamente cuatro veces menos luego de 41 días desde la última suplementación, mientras que otros autores han planteado que intervenciones crónicas podrían mejorar la recuperación muscular, no así las adaptaciones propias al entrenamiento deportivo $(44,45)$.

Los resultados de esta revisión no permiten determinar un efecto real asociado al consumo de vitamina $\mathrm{E}$ y disminución del DOMS; es poco probable que las pequeñas diferencias estadísticas en la percepción del DOMS que favorecen la suplementación con vitamina $E$ equivalgan a diferencias importantes en la práctica, debido, 
sobre todo, a los escasos estudios que analizan la suplementación de vitamina $E$ en forma única.

Finalmente, la literatura recomienda, para prevenir el daño oxidativo y mejorar la respuesta inmune, dosis entre 5 y 19 mg/día para poblaciones sedentarias y entre 100 y $200 \mathrm{mg} /$ día para deportistas de resistencia aeróbica; sin embargo, estos valores pueden oscilar dependiendo de factores como rango etario, sexo, zona geográfica de residencia y déficit nutricional, siendo este último bastante raro en seres humanos $(11,46-50)$.

\section{CONCLUSIÓN}

La suplementación con vitamina $E$ produce una disminución del estrés oxidativo y estabilización del sarcolema, aunque faltan pruebas concluyentes para afirmar que la vitamina $\mathrm{E}$ puede mejorar en los biomarcadores asociados al DOMS.

\section{DECLARACIÓN DE CONFLICTO DE INTERESES}

Los autores declaran que no existen conflictos de intereses.

\section{- -Referencias}

1. Ortiz Rodríguez B, Carrasco-Legleu CE, De León Fierro LG, Candia-Sosa KF, Candia-Lujan R, Najera Longoria RJ. Suplementos nutricionales en el tratamiento y la prevención del dolor muscular tardío: una revisión sistemática. Retos. 2019;35:407-12 Disponible en: https://recyt.fecyt.es/index.php/retos/article/view/61908

2. Candia-Luján $\mathrm{R}, \mathrm{De}$ Paz Fernández JA, Costa Moreira $\mathrm{O}$. Are antioxidant supplements effective in reducing delayed onset muscle soreness? A systematic review. Nutr Hosp. 2015;31(1):32-45. https://doi.org/10.3305/nh.2015.31.1.8171

3. Candia-Luján R, De Paz Fernández JA, Costa Moreira O. ¿Son efectivos los antiinflamatorios no esteroides en el tratamiento del dolor muscular tardío? Ciencia UAT. 2014;9(1):76-83. https://doi.org/10.29059/cienciauat.v9i1.630

4. Xu J, Fu SN, Zhou D, Huang C, Hug F. Relationship between pre-exercise muscle stiffness and muscle damage induced by eccentric exercise. Eur J Sport Sci. 2018;26:1-9. https://doi.org/10.1080/17461391.2018.1535625

5. Hotfiel T, Freiwald J, Hoppe MW, Lutter C, Forst R, Grim C, et al. Advances in delayed-onset muscle soreness (DOMS). Part l: Pathogenesis and diagnostics. Sportverletz Sportschaden. 2018;32(4):243-50. https://doi.org/10.1055/a-0753-1884

6. Rahimi MH, Mohammadi H, Eshaghi H, Askari G, Miraghajani M. The effects of beta-hydroxy-beta-methylbutyrate supplementation on recovery following exercise-induced muscle damage: A systematic review and meta-analysis. J Am Coll Nutr. 2018;37(7):640-49. https://doi.org/10.1080/07315724.2018.1451789

7. Rahimi MH, Shab-Bidar S, Mollahosseini M, Djafarian K. Branched-chain amino acid supplementation and exerciseinduced muscle damage in exercise recovery: A meta-analysis of randomized clinical trials. Nutrition. 2017;42:30-36. https://doi.org/10.1016/.nut.2017.05.005

8. Bloomer RJ. The role of nutritional supplements in the prevention and treatment of resistance exercise-induced skeletal muscle injury. Sports Med. 2007;37(6):519-32. https://doi.org/10.2165/00007256-200737060-00005

9. Alcantara Montero, A. Vitamina D y dolor crónico. Rev. Soc. Esp. Dolor. 2016;23(4):211-4. https://doi.org/10.20986/resed.2016.3429/2016

10. Heiss R, Lutter C, Freiwald J, Hoppe MW, Grim C, Poettgen K, et al. Advances in delayed-onset muscle soreness (DOMS). Part II: Treatment and prevention. Sportverletz Sportschaden. 2019;33(1):21-9. https://doi.org/10.1055/a-0810-3516 
11. Péter S, Friedel A, Roos FF, Wyss A, Eggersdorfer M, Hoffmann K, et al. A systematic review of global alpha-tocopherol status as assessed by nutritional intake levels and blood serum concentrations. Int J Vitam Nutr Res. 2015;85(5-6):261-81. https://doi.org/10.1024/0300-9831/a000281

12. Niki E, Traber MG. A history of vitamin E. Ann Nutr Metab. 2012;61(3):207-12. https://doi.org/10.1159/000343106

13. Febles C, Soto C, Saldaña A, García B. Funciones de la vitamina E: Actualización. Rev Cubana Estomatol. 2002;39(1):28-32. Disponible en: http://scielo.sld.cu/scielo.php?script=sci_arttext\&pid=S003475072002000100005\&lng=es

14. Sacheck JM, Blumberg JB. Role of vitamin E and oxidative stress in exercise. Nutrition. 2001;17(10):809-14. https://doi. org/10.1016/S0899-9007(01)00639-6

15. He F, Hockemeyer JA, Sedlock D. Does combined antioxidant vitamin supplementation blunt repeated bout effect? Int J Sports Med. 2015;36(5):407-13. https://doi.org/10.1055/s-0034-1395630

16. Chou CC, Sung YC, Davison G, Chen CY, Liao YH. Short-Term high-dose vitamin C and E supplementation attenuates muscle damage and inflammatory responses to repeated taekwondo competitions: A randomized placebo-controlled trial. Int J Med Sci. 2018;15(11):1217-26. https://doi.org/10.7150/ijms.26340

17. Howatson G, van Someren KA. The prevention and treatment of exercise-induced muscle damage. Sports Med. 2008;38(6):483-503. https://doi.org/10.2165/00007256-200838060-00004

18. Gaeini AA, Rahnama N, Hamedinia, MR. Effects of vitamin E supplementation on oxidative stress at rest and after exercise to exhaustion in athletic students. J Sports Med Phys Fitness 2006;46(3):458-61.

19. Shafat $A$, Butler $P$, Jensen $R L$, Donnelly $A E$. Effects of dietary supplementation with vitamins $C$ and $E$ on muscle function during and after eccentric contractions in humans. European Journal of Applied Physiology 2004;93(1-2):196-202. https://doi.org/10.1007/s00421-004-1198-y

20. Theodorou AA, Nikolaidis MG, Paschalis V, Koutsias S, Panayiotou G, Fatouros IG, et al. No effect of antioxidant supplementation on muscle performance and blood redox status adaptations to eccentric training. American Journal of Clinical Nutrition 2011;93(6):1373-83. https://doi.org/10.3945/ajcn.110.009266

21. Morrison D, Hughes J, Della Gatta PA, Mason S, Lamon S, Russell AP, Wadley GD. Vitamin C and E supplementation prevents some of the cellular adaptations to endurance-training in humans. Free Radic Biol Med. 2015;89:852-62. https://doi.org/10.1016/j.freeradbiomed.2015.10.412

22. Ranchordas MK, Rogerson D, Soltani H, Costello JT. Antioxidants for preventing and reducing muscle soreness after exercise. Cochrane Database Syst Rev. 2017;12:CD009789. https://doi.org/10.1002/14651858.CD009789.pub2

23. Silva LA, Pinho CA, Silveira PC, Tuon T, De Souza CT, Dal-Pizzol F, et al. Vitamin E supplementation decreases muscular and oxidative damage but not inflammatory response induced by eccentric contraction. J Physiol Sci. 2010;60(1):51-7. https://doi.org/10.1007/s12576-009-0065-3

24. Avery N.G, Kaiser J.L, Sharman M.J, Scheett T.P, Barnes D.M., Gómez A.L. et al. Effects of vitamin E supplementation on recovery from repeated bouts of resistance exercise. J. Strength Cond. Res. 2003:17:801-809. https://doi.org/10.1519/00124278200311000-00028

25. Beaton LJ, Allan DA, Tarnopolsky MA, Tiidus PM, Phillips SM. Contraction-induced muscle damage is unaffected by vitamin E supplementation. Med Sci Sports Exerc. 2002;34(5):798-805. https://doi.org/10.1097/00005768-200205000-00012

26. Itoh, H., Ohkuwa, T., Yamazaki, Y., Shimoda, T., Wakayama, A., Tamura, S., et al. Vitamin E supplementation attenuates leakage of enzymes following 6 successive days of running training. Int. J. Sports Med. 2000;21:369-74. https://doi.org/10.1055/s-2000-3777 


\section{Efectos de la suplementación con vitamina E}

27. Sacheck, J.M, Milbury, P.E, Cannon J.G, Roubenoff R., Blumberg J.B. Effect of vitamin E and eccentric exercise on selected biomarkers of oxidative stress in young and elderly men. Free Radical Biol. Med. 2003;34(12):1575-88. https://doi.org/10.1016/S0891-5849(03)00187-4

28. Santos SA, Silva ET, Caris AV, Lira FS, Tufik S, Dos Santos RV. Vitamin E supplementation inhibits muscle damage and inflammation after moderate exercise in hypoxia. J Hum Nutr Diet. 2016;29(4):516-22. https://doi.org/10.1111/jhn.12361

29. Palacios P, Pedrero-Chamizo R, Palacios N, Maroto-Sánchez B, Aznar S, González-Gross M. Biomarcadores de la actividad física y del deporte. Rev Esp Nutr Comunitaria 2015;21(Supl.1):235-42. https://doi.org/10.14642/RENC.2015.21.sup1.5070

30. Fernándeza JM, Da Silva-Grigolettob ME, Túnez-Fiñana I. Estrés oxidativo inducido por el ejercicio. Rev Andal Med Deporte. 2009;2(1):19-34. Disponible en: https://www.elsevier.es/es-revista-revista-andaluza-medicina-del-deporte-284-pdf-13134195

31. Sies H. Oxidative stress: a concept in redox biology and medicine. Redox Biol. 2015;4:180-3. https://doi.org/10.1016/.redox.2015.01.002

32. Powers SK, Radak Z, Ji LL. Exercise-induced oxidative stress: past, present and future. J Physiol. 2016;594(18):5081-92. https://doi.org/10.1113/JP270646

33. Powers SK, Jackson MJ. Exercise-induced oxidative stress: cellular mechanisms and impact on muscle force production. Physiol Rev. 2008;88(4):1243-76. https://doi.org/10.1152/physrev.00031.2007

34. Venditti P, Napolitano G, Barone D, Di Meo S. Vitamin E supplementation modifies adaptive responses to training in rat skeletal muscle. Free Radic Res. 2014;48(10):1179-89. https://doi.org/10.3109/10715762.2014.937341

35. Niki E. Evidence for beneficial effects of vitamin E. Korean J Intern Med. 2015;30(5):571-9. https://doi.org/10.3904/ kjim.2015.30.5.571

36. Stepanyan V, Crowe M, Haleagrahara N, Bowden B. Effects of vitamin E supplementation on exercise-induced oxidative stress: a meta-analysis. Appl Physiol Nutr Metab. 2014;39(9):1029-37. https://doi.org/10.1139/apnm-2013-0566

37. Chung E, Mo H, Wang S, Zu Y, Elfakhani M, Rios SR, et al. Potential roles of vitamin E in age-related changes in skeletal muscle health. Nutr Res. 2018;49:23-36. https://doi.org/10.1016/.nutres.2017.09.005

38. Salehi B, Martorell M, Arbiser JL, Sureda A, Martins N, Maurya PK, et al. Antioxidants: Positive or Negative Actors? Biomolecules. 2018;8(4):124. https://doi.org/10.3390/biom8040124

39. de Oliveira DCX, Rosa FT, Simões-Ambrósio L, Jordao AA, Deminice R. Antioxidant vitamin supplementation prevents oxidative stress but does not enhance performance in young football athletes. Nutrition. 2019;63-64:29-35. https://doi.org/10.1016/j.nut.2019.01.007

40. Kyparos A, Nikolaidis MG, Dipla K, Zafeiridis A, Paschalis V, Vrabas IS. Low-frequency fatigue as an indicator of eccentric exercise-induced muscle injury: The role of Vitamin E. Oxid Med Cell Longev. 2012;628352. https://doi.org/10.1155/2012/628352

41. Peake, J.M., Suzuki, K., and Coombes, J.S. The influence of antioxidant supplementation on markers of inflammation and the relationship to oxidative stress after exercise. J. Nutr. Biochem. 2007;18(6):357-71. https://doi.org/10.1016/j.jnutbio.2006.10.005

42. McGinley C, Shafat A., Donnelly A.E. Does antioxidant vitamin supplementation protect against muscle damage? Sports Med. 2009;39(12):1011-32. https://doi.org/10.2165/11317890-000000000-00000

43. Burton GW, Traber MG., Acuff RV, Walters DN, Kayden H, Hughes L, et al. Human plasma and tissue -tocopherol concentrations in response to supplementation with deuterated natural and synthetic vitamin E. Am J Clin Nutr. 1998:67(4):669-84. https://doi.org/10.1093/ajcn/67.4.669 
44. Mastaloudis A, Traber MG, Carstensen K, Widrick JJ. Antioxidants did not prevent muscle damage in response to an ultramarathon run. Med Sci Sports Exerc. 2006;38(1):72-80. https://doi.org/10.1249/01.mss.0000188579.36272.f6

45. Fisher-Wellman K, Bloomer RJ. Acute exercise and oxidative stress: a 30-year history. Dyn. Med. 2009;8:1-25. https://doi. org/10.1186/1476-5918-8-1

46. Institute of Medicine (US). Dietary Reference Intakes for Vitamin C, Vitamin E, Selenium, and Carotenoids. Washington DC: National Academies Press (US); 2000.

47. Scientific Committee on Food (SCF). Opinion of the SCF on the revision of reference values. for nutrition labelling. 2003. Disponible en: https://www.nutri-facts.org/content/dam/nutrifacts/pdt/nutrients/SCF_Reference_values_for_nutrition_2003.pdf

48. Takanami Y, Iwane H, Kawai Y, Shimomitsu T. Vitamin E supplementation and endurance exercise: are there benefits?. Sports Med. 2000;29(2):73-83. https://doi.org/10.2165/00007256-200029020-00001

49. Kemnic TR, Coleman M. Vitamin E Deficiency. Treasure Island: StatPearls [Internet]. [Citado enero de 2020]. Disponible en: https:// www.ncbi.nlm.nih.gov/books/NBK519051/

50. Lee GY, Han SN. The role of vitamin E in immunity. Nutrients. 2018;10(11). pii:E1614. https://doi.org/10.3390/nu10111614 